\title{
Attitudes to Bystander Cardiopulmonary Resuscitation in Japan in 2010
}

\author{
Takumi Taniguchi, MD; Koji Sato, MD; Takashi Fujita, MD; \\ Masaki Okajima, MD; Masayuki Takamura, MD
}

\begin{abstract}
Background: Early initiation of bystander cardiopulmonary resuscitation (CPR) improves the chances of successful resuscitation and survival. The aim of the present study was to identify the attitudes of Japanese subjects toward bystander CPR and to compare them with those observed in previous studies in 1998 and 2006.

Methods and Results: Participants were asked about their willingness to perform CPR in 5 different scenarios and their willingness to perform chest compression (CC) plus mouth-to-mouth ventilation (MMV) versus $C C$ alone. A total of 2,785 individuals completed the questionnaire, including high school students, teachers, medical nurses, and medical students, whose characteristics were not statistically different from those in the previous studies. Only 15$30 \%$ of participants were likely to perform CC plus MMV, especially on a stranger or a trauma victim; these percentages in nurses and medical students were significantly lower than those in the previous studies. But $50-100 \%$ of them were likely to perform CC alone, consistent with the results obtained in 2006 . The reasons for the unwillingness among laypeople to perform CC plus MMV were inadequate knowledge and/or doubt regarding whether they could perform the techniques effectively, while health-care providers reported a fear of disease transmission.
\end{abstract}

Conclusions: Most participants are unlikely to perform CC plus MMV, especially on a stranger or trauma victim, but are more likely to perform CC alone, as also found in the previous studies. (Circ $J$ 2012; 76: 1130-1135)

Key Words: Bystander CPR; Cardiopulmonary resuscitation; Chest compression; Mouth-to-mouth ventilation

$\mathbf{E}$ arly initiation of bystander cardiopulmonary resuscitation (CPR) improves the chances of successful resuscitation and survival..$^{1,2}$ There is increasing awareness of the importance of bystander CPR, and there has been an increase in attendance at CPR training courses in the USA, Europe, and Japan. The guidelines published by the American Heart Association in 2005 recommended chest compression (CC) and mouth-to-mouth ventilation (MMV) as the best method of CPR. ${ }^{3}$ In 2006, however, we showed that most laypeople and health-care providers are unlikely to perform CC plus MMV, especially on a stranger or trauma victim, but are more likely to perform $\mathrm{CC}$ alone, consistent with the results of a previous study in $1998 .{ }^{4}$ Moreover, several studies have indicated that the need for mouth-to-mouth contact discourages not only laypeople but also health-care providers from performing bystander CPR. ${ }^{5-8}$ Few reports have been published, however, on attitudes to bystander CPR, therefore, the aims of this study were to identify the attitudes of Japanese people toward the performance of bystander CPR, in order to compare the findings with those obtained in previous studies in 1998 and 2006.

\section{Methods}

Data were collected in accordance with the national guideline of ethics for epidemiological survey (Ministry of Health, Labor and Welfare in Japan). The study was approved by the institutional review board of Kanazawa University School of Medicine.

\section{Respondent Demographics}

The respondents were inhabitants of Ishikawa, Japan, a prefecture with a mixture of urban and rural environments and a resident population of $>1.1$ million, which was the same as in the previous studies. ${ }^{4,9}$ The respondents were high school students, high school teachers, medical nurses, and medical students. All respondents were given a written questionnaire. The high school students and teachers were chosen from 12 high schools evaluated in the previous studies. The respondents were chosen by the principals of the schools. The nurses represented the entire staff of full-time nurses working at an 850-bed university-affiliated teaching hospital and a 200-bed municipal hospital, which were also evaluated in the previous

Received January 18, 2011; revised manuscript received January 13, 2012; accepted January 16, 2012; released online March 2 , 2012 Time for primary review: 37 days

Intensive Care Unit, Kanazawa University Hospital, Kanazawa (T.T., K.S., T.F., M.O.); Department of Disease Control and Homeostasis, Graduate School of Medicine, Kanazawa University, Kanazawa (M.T.), Japan

Mailing address: Takumi Taniguchi, MD, Intensive Care Unit, Kanazawa University Hospital, 13-1 Takara-machi, Kanazawa 920-8641, Japan. E-mail: takutaniyan@yahoo.co.jp

ISSN-1346-9843 doi:10.1253/circj.CJ-11-0054

All rights are reserved to the Japanese Circulation Society. For permissions, please e-mail: cj@j-circ.or.jp 


\section{Table 1. List of Scenarios}

\section{Would you perform CPR on someone you had never seen before?}

[Scenario] You are taking a walk. The man walking in front of you has cardiopulmonary arrest. You did not get a good look at him, but he was between the age of 30 and 50 years old, not disheveled, but definitely not well dressed. You summon for help. Then would you ... ?

\section{Would you perform CPR on a trauma victim?}

[Scenario] It is in the morning rush hour and you witness a motor vehicle accident. You run to the scene to render assistance. There is 1 person in the car, the driver, who is a 30 -year-old man, well dressed. He has suffered a cardiopulmonary arrest, and there is a small amount of blood dripping from his forehead onto his face. You summon for help. Then, would you ...?

\section{Would you perform CPR on a child?}

[Scenario] It is a holiday and a child knocks on your door, points to her friend, and says 'I think something is wrong with my friend.' They are both about 6 years old. Her friend suddenly collapses in front of you with cardiopulmonary arrest. You summon for help. Then, would you ... ?

\section{Would you perform CPR on an elderly person?}

[Scenario] You are visiting a patient in a nursing home who you have taken care of before. Suddenly, a nurse at this nursing home asks you to take a look at another patient. You comply, and this 70 -year-old, previously healthyappearing patient has suffered a cardiopulmonary arrest. You summon for help. Then, would you ... ?

\section{Would you perform CPR on a relative?}

[Scenario] Your grandmother is taking a bath and has a cardiopulmonary arrest in the bathtub. She is 72 years old and previously has been healthy. You summon for help. Then, would you ... ?

CPR, cardiopulmonary resuscitation.

studies. The medical students were in the fourth year of a 6year program in the hospital's School of Medicine. The nurses and medical students were volunteers. All high schools and hospitals in the present study were the same used in the previous studies in 1998 and 2006.

\section{Questionnaires}

Respondents to the questionnaire were presented with 5 hypothetical scenarios of cardiopulmonary arrest (Table 1), as in the previous studies, ${ }^{4,9}$ and were surveyed from March to April 2010. They were asked to assume that no one else was available to help. In addition, health-care providers (medical nurses, and medical students) were asked to respond in their non-professional capacity, that is, as ordinary citizens. Options for rescue response included delivery of full CPR (CC plus MMV), $\mathrm{CC}$ alone, or no active intervention except summoning help. Respondents indicating willingness to perform $\mathrm{CC}$ alone or no direct intervention were defined as refusing MMV. Respondents were instructed to choose the option that they thought best characterized what they would do if actually faced with the situation. If they indicated willingness to perform CC alone or no direct intervention, respondents were asked to choose from the following reasons for not performing MMV: poor knowledge; poor skills; fear of disease transmission; unwillingness to touch the victim; fear of hurting the patient; and others.

\section{Data Analysis}

The influence of the CPR technique involved and previous CPR training on the respondents' willingness to perform CPR was analyzed using Fisher's exact test and 1-way analysis of variance (ANOVA) on ranks. When ANOVA detected a significant difference, post hoc testing was performed using Dunn's method. The level of statistical significance was set at $\mathrm{P}<0.05$.

\section{Results}

\section{Respondents}

Of the 810 nurses, $748(92.3 \%)$, and of the 101 of medical students, $91(90.1 \%)$ provided completed questionnaires.

\begin{tabular}{|c|c|c|c|c|}
\hline & 2010 & 2006 & 1998 & $P$ value \\
\hline \multicolumn{5}{|c|}{ High school students } \\
\hline $\mathrm{n}$ & 1,708 & 3,125 & 479 & \\
\hline First grade & 27 & 30 & 28 & 0.642 \\
\hline Second grade & 68 & 66 & 65 & 0.774 \\
\hline Third grade & 5 & 4 & 7 & 0.345 \\
\hline Male & 53 & 51 & 41 & 0.221 \\
\hline \multicolumn{5}{|c|}{ High school teachers } \\
\hline $\mathrm{n}$ & 238 & 319 & 147 & \\
\hline Age (years) & $44 \pm 12$ & $42 \pm 12$ & $34 \pm 11$ & 0.264 \\
\hline Male & 63 & 61 & 69 & 0.188 \\
\hline \multicolumn{5}{|l|}{ Medical nurses } \\
\hline $\mathrm{n}$ & 748 & 451 & 424 & \\
\hline Age (years) & $34 \pm 7$ & $32 \pm 8$ & $34 \pm 8$ & 0.688 \\
\hline Male & 3 & 3 & 3 & 0.884 \\
\hline \multicolumn{5}{|l|}{ Medical students } \\
\hline $\mathrm{n}$ & 91 & 179 & 104 & \\
\hline Age (years) & $22 \pm 1$ & $22 \pm 2$ & $24 \pm 2$ & 0.892 \\
\hline Male & 69 & 79 & 85 & 0.098 \\
\hline
\end{tabular}

Data given as $\%$ or mean \pm SD.

Mean age of the nurses and medical students was 34 and 22 years old, respectively, and there were no significantly differences between the present study and the previous studies (Table 2). Among high school students, 27\% were in the first year, $68 \%$ in the second year, and 5\% in the last year of a 3year program, and there were no significant differences between the present study and the previous studies. Moreover, no significant differences were noted with regard to sex of each respondent category between the present study and the previous studies.

Approximately $70 \%$ of respondents had received CPR training more than once (Table 3). There was no significant difference between the number of previous CPR training courses taken by the high school students in the present study and that in the previous studies. In contrast, the number of 


\begin{tabular}{|lcccc|}
\hline Table 3. Previous CPR Training of $\mathbf{2 0 1 0}$ Respondents \\
\cline { 2 - 5 } & None & Once & Twice & $>\mathbf{3}$ times \\
High school students & $43(41 / 32)$ & $33^{*}(38 / 40)$ & $14(15 / 20)$ & $10^{*}(6 / 8)$ \\
High school teachers & $6^{*}(15 / 60)$ & $21(25 / 20)$ & $33^{*}(24 / 11)$ & $40^{*}(35 / 9)$ \\
Medical nurses & $2(4 / 5)$ & $25(27 / 27)$ & $33(32 / 40)$ & $40^{*}(37 / 28)$ \\
Medical students & $1(1 / 5)$ & $7^{*}(19 / 54)$ & $28^{*}(39 / 30)$ & $65^{\star}(41 / 11)$ \\
\hline
\end{tabular}

Data given as $n(\%) .{ }^{*} P<0.05$ vs. previous studies in 2006 and 1998 .

CPR, cardiopulmonary resuscitation.

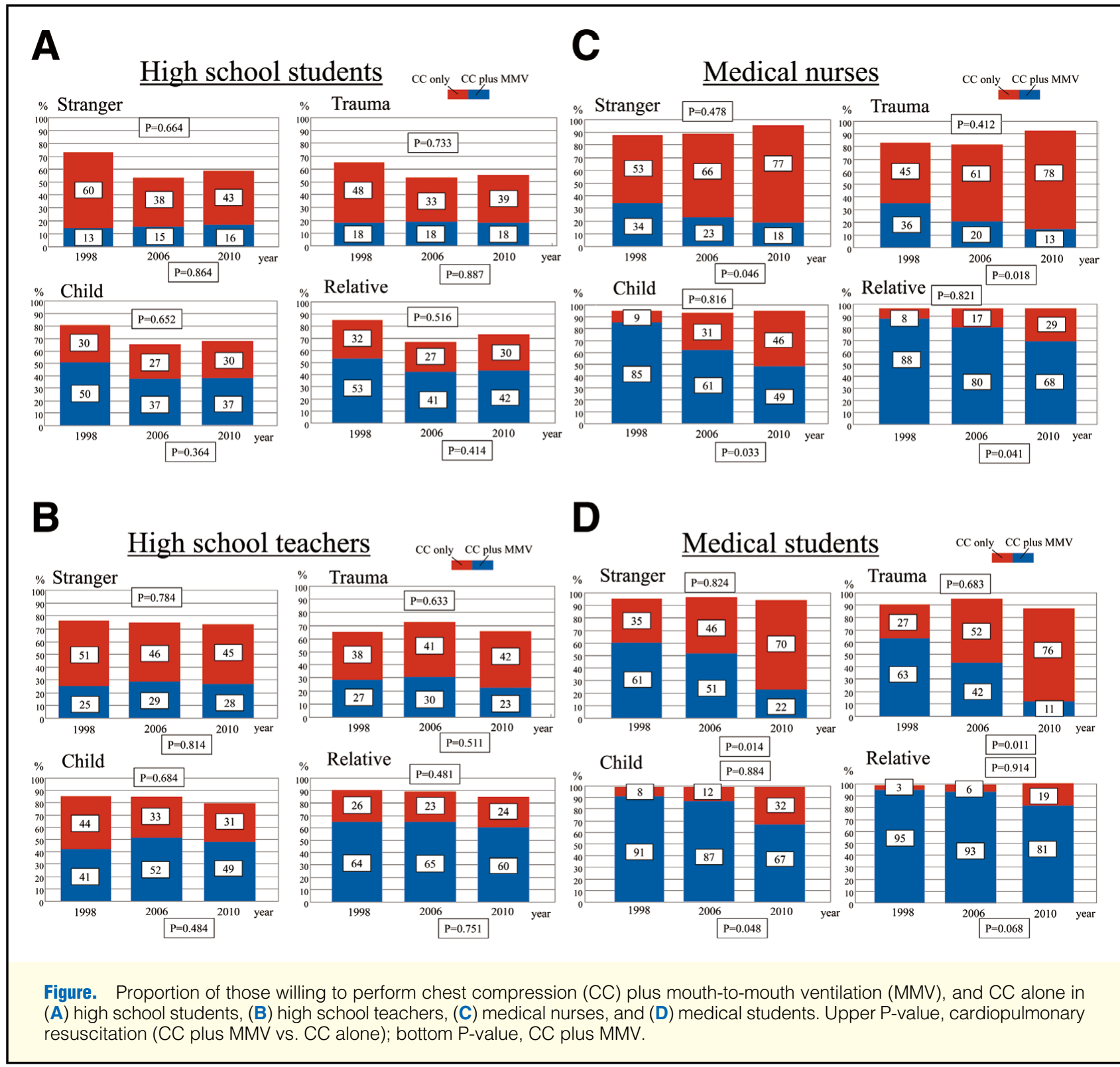

previous CPR training courses taken by the high school teachers was significantly higher than in the previous studies. In all groups, the prevalence of previous CPR training more than 3 times was significantly higher than in the previous studies.

\section{Responses}

In all respondent categories, willingness to perform CPR (CC plus MMV and CC alone) was low for a stranger or a trauma victim with blood on the face (scenarios 1 and 2), but high for a child or a relative (scenarios 3 and 5; Figure). Respondent age or gender had no influence on willingness in any group. 


\begin{tabular}{|c|c|c|c|c|}
\hline Scenario & $\begin{array}{l}\text { High school } \\
\text { students (\%) }\end{array}$ & $\begin{array}{l}\text { High school } \\
\text { teachers (\%) }\end{array}$ & Nurses (\%) & $\begin{array}{c}\text { Medical } \\
\text { students (\%) }\end{array}$ \\
\hline \multicolumn{5}{|l|}{ Stranger } \\
\hline Fear of incomplete performance or poor knowledge & 67 & 56 & 9 & 1 \\
\hline Fear of hurting a victim & 14 & 8 & 0 & 0 \\
\hline Fear of disease transmission & 7 & 23 & 53 & 63 \\
\hline $\mathrm{CC}$ alone & 1 & 5 & 15 & 10 \\
\hline Others & 11 & 8 & 12 & 16 \\
\hline Unknown & 12 & 15 & 18 & 18 \\
\hline \multicolumn{5}{|l|}{ Trauma victim } \\
\hline Fear of incomplete performance or poor knowledge & 58 & 62 & 11 & 3 \\
\hline Fear of hurting a victim & 18 & 12 & 3 & 3 \\
\hline Fear of disease transmission & 27 & 38 & 83 & 82 \\
\hline $\mathrm{CC}$ alone & 3 & 8 & 19 & 16 \\
\hline Others & 14 & 11 & 16 & 18 \\
\hline Unknown & 15 & 18 & 21 & 20 \\
\hline \multicolumn{5}{|l|}{ Child } \\
\hline Fear of incomplete performance or poor knowledge & 77 & 71 & 15 & 1 \\
\hline Fear of hurting a victim & 20 & 7 & 9 & 0 \\
\hline Fear of disease transmission & 3 & 7 & 35 & 61 \\
\hline $\mathrm{CC}$ alone & 0 & 9 & 9 & 3 \\
\hline Others & 6 & 10 & 21 & 18 \\
\hline Unknown & 12 & 12 & 28 & 25 \\
\hline \multicolumn{5}{|l|}{ Relative } \\
\hline Fear of incomplete performance or poor knowledge & 72 & 68 & 14 & 4 \\
\hline Fear of hurting a victim & 21 & 11 & 3 & 0 \\
\hline Fear of disease transmission & 6 & 16 & 26 & 28 \\
\hline $\mathrm{CC}$ alone & 2 & 8 & 37 & 35 \\
\hline Others & 14 & 11 & 18 & 21 \\
\hline Unknown & 16 & 17 & 21 & 26 \\
\hline
\end{tabular}

$\mathrm{CC}$, chest compression; MMV, mouth-to-mouth ventilation.

High school students and teachers were similar in their level of willingness, and medical nurses and medical students were almost identical in their level of willingness to perform $\mathrm{CC}$ plus MMV.

In all respondent categories, willingness to perform $\mathrm{CC}$ plus MMV was low for all scenarios, especially for a stranger or a trauma victim with blood on the face. Only $15-25 \%$ of respondents in all categories reported willingness to perform $\mathrm{CC}$ plus MMV in these scenarios.

\section{Comparison With Previous Studies}

In all respondent categories, there was no significant difference in willingness to perform CPR (CC plus MMV and CC alone) between the present study and the previous studies for any of the scenarios included (Figure). Medical nurses and medical students showed a significantly lower degree of willingness to perform CC plus MMV in all scenarios except for the scenario regarding the relative, compared to the previous studies.

\section{Reasons for Not Performing CC Plus MMV}

In the scenario regarding the stranger, among high school students, only $7 \%$ of those who declined to perform CC plus MMV stated that they would not do so because of concern about disease transmission, while $67 \%$ claimed that they declined because of poor knowledge and/or fear of imperfect performance of CC plus MMV. These findings were similar to those in the previous studies. Among high school teachers, $23 \%$ of those who declined to perform CC plus MMV stated that they would not do so because of concern about disease transmission. This percentage was significantly higher than in the previous studies. In the scenarios for trauma victim and relative, among high school students and teachers, reasons for not performing CC plus MMV were similar to those in the previous studies.

In the scenario regarding the stranger, approximately 50$70 \%$ of medical nurses and students who declined to perform CC plus MMV refused because of fear of disease transmission. Among medical nurses and medical students, the degree of concern about disease transmission was similar to that in the previous studies. In the scenarios for trauma victim and relative, reasons for not performing CC plus MMV were similar to those in the previous studies.

In the child scenario, among high school students and teachers, only $3-7 \%$ of those who declined to perform CC plus MMV stated that they would not do so because of concern about disease transmission, while $>70 \%$ claimed that they declined because of poor knowledge and/or fear of imperfect performance of CC plus MMV (Table 4). The reasons for not performing CC plus MMV in the present study were not significantly different from the previous studies. Among medical nurses and medical students, the degree of concern about disease transmission was similar to that in the previous studies. 


\section{Discussion}

Only $15-30 \%$ of laypeople and health-care providers were likely to perform CC plus MMV, especially on a stranger or a trauma victim with blood on the face; these proportions of nurses and medical students were significantly lower than that obtained in the studies performed in 1998 and 2006. But 50$100 \%$ of them were likely to perform CC alone, which was consistent with the results obtained in 2006. The reasons for unwillingness among high school students and teachers to perform CC plus MMV were inadequate knowledge and/or doubt about their capability of performing CC plus MMV, while the main reason among nurses and medical students was the fear of disease transmission, which was consistent with that in the previous studies.

The present study shows that the number of previous CPR training courses taken by the high school students in the present study was not significantly different from those in the previous studies, although the number of previous CPR training courses taken by people in other categories was higher than that in the previous studies. Moreover, the present study shows that Japanese high school students were reluctant to perform $\mathrm{CC}$ alone in all scenarios, consistent with the previous results, and that the reasons for unwillingness to perform $\mathrm{CC}$ plus MMV among high school students were their poor knowledge and/or fear of inadequate performance, which is similar to the reasons reported in the previous studies. These findings suggest that it may be possible that high school students would perform CC plus MMV or CC alone if they took repeated and more effective CPR training courses.

The present study also shows that the number of previous CPR training courses taken by high school teachers was significantly higher than that reported in the previous studies. This finding suggests that high school teachers may be more interested in learning about prevention of cardiac arrest. High school teachers, however, were also reluctant to perform CC plus MMV on strangers and trauma victims, and a major reason for the unwillingness was their poor knowledge and/or lack of ability to perform CC plus MMV, as in the previous studies. Moreover, the proportions of those willing to perform $\mathrm{CC}$ alone in the scenarios of trauma, child and relative did not differ from those in the previous study. These findings suggest that the CPR training courses taken may not be effective and that the training courses did not emphasize CC alone. Therefore, it may be necessary to re-evaluate CPR training programs.

Nurses and medical students were more reluctant to perform CC plus MMV in all scenarios than in the previous studies, although the number of previous CPR training courses taken by these subjects was significantly greater than those reported in the previous studies. In contrast, nurses and medical students were not reluctant to perform $\mathrm{CC}$ alone in all scenarios, as in the previous studies. Several recent studies have demonstrated that CPR using $\mathrm{CC}$ alone is the preferable approach, or is at least similar in effectiveness to CPR with CC plus MMV, for adult patients experiencing an out-of-hospital cardiac arrest. ${ }^{10-12}$ These findings suggest that nurses and medical students, as well as laypeople, should be encouraged to perform $\mathrm{CC}$ alone if they are unwilling to provide rescue breathing.

The difference in the proportion of people reluctant to perform CC plus MMV between laypeople and health-care providers is interesting. The number of those willing to perform CC plus MMV in laypeople did not differ from that in the previous study. In nurses and medical students, however, the number of those willing to perform CC plus MMV in all scenarios was significantly lower than in the previous study. These findings suggest that the CPR training courses taken in the different respondent categories may be different and/or may not be effective. In particular, the present study did not evaluate the CPR training courses that high school students and teachers had taken. Further investigation is needed to evaluate the CPR training courses and teaching among the respondent categories.

In the child scenario, the number of people willing to perform CC plus MMV among the nurses and medical students was significantly lower than that in the previous study. One of the major reasons for the unwillingness was due to the awareness that $\mathrm{CC}$ alone is the preferred resuscitation method for children. These findings have very important implications for CPR training for children. Several studies have demonstrated that CC plus MMV by bystanders is associated with higher survival than $\mathrm{CC}$ alone in children. ${ }^{13,14}$ It may be necessary to re-evaluate CPR training programs for children.

In the present study, the scenarios of cardiopulmonary arrest were similar and the respondent categories were the same, and the schools and hospitals were also the same as in the previous studies in order to achieve an accurate comparison. Moreover, it is possible that some of the high school teachers and nurses in the present study also took part in the previous studies, but it was not possible to clarify this further. Further investigation is needed to identify Japanese attitudes toward the performance of bystander CPR.

In summary, most laypeople and health-care providers are unlikely to perform CC plus MMV, but are more likely to perform CC only, as also found in the previous studies in 1998 and 2006. These findings suggest that MMV training should be de-emphasized and the awareness of $\mathrm{CC}$ alone should be emphasized. The CPR training program for children, however, may need re-evaluation because of the importance of MMV in children.

\section{References}

1. Gallagher EJ, Lombardi G, Gennis P. Effectiveness of bystander cardiopulmonary resuscitation and survival following out-of-hospital cardiac arrest. JAMA 1995; 274: 1922-1925.

2. Kida M, Kawamura T, Fukuoka T, Tamakoshi A, Wakai K, Ohno Y, et al. Out-of-hospital cardiac arrest and survival: An epidemiological analysis of emergency service reports in a large city in Japan. Circ J 2004; 68: 603-609.

3. 2005 American Heart Association guidelines for cardiopulmonary resuscitation and emergency cardiovascular care. Circulation 2005; 112: IV19-IV33.

4. Taniguchi T, Omi W, Inaba H. Attitudes toward the performance of bystander cardiopulmonary resuscitation in Japan. Resuscitation 2007; 75: $82-87$.

5. Cho GC, Sohn YD, Kang KH, Lee WW, Lim KS, Kim W, et al. The effect of basic life support education on laypersons' willingness in performing bystander hands only cardiopulmonary resuscitation. Resuscitation 2010; 81: 691-694.

6. Hamasu S, Morimoto T, Kuramoto N, Horiguchi M, Iwami T, Nishiyama C, et al. Effects of BLS training on factors associated with attitude toward CPR in college students. Resuscitation 2009; 80: 359-364.

7. Hew P, Brenner B, Kaufman J. Reluctance of paramedics and emergency medical technicians to perform mouth-to-mouth resuscitation. J Emerg Med 1997; 15: 279-284.

8. Yokoyama H, Yonemoto N, Yonezawa K, Fuse J, Shimizu N, Hayashi T, et al. Report from the Japanese registry of CPR for inhospital cardiac arrest (J-RCPR). Circ J 2011; 75: 815-822.

9. Shibata K, Taniguchi T, Yoshida M, Yamamoto K. Obstacles to bystander cardiopulmonary resuscitation in Japan. Resuscitation 2000; 44: $187-193$.

10. SOS-KANTO study group. Cardiopulmonary resuscitation by bystanders with chest compression only (SOS-KANTO): An observa- 
tional study. Lancet 2007; 369: 920-926.

11. Iwami T, Kawamura T, Hiraide A, Berg RA, Hayashi Y, Nishiuchi $\mathrm{T}$, et al. Effectiveness of bystander-initiated cardiac-only resuscitation for patients with out-of-hospital cardiac arrest. Circulation 2007; 116: $2900-2907$.

12. Nakagawa Y, Inokuchi S, Morita S, Akieda K, Kaji A, Komine T, et al. Accuracy of pulse checks in terms of basic life support by lifesavers, as laypersons. Circ J 2010; 74: 1895-1899.

13. Kitamura T, Iwami T, Kawamura T, Nagao K, Tanaka H, Nadkarni
VM, et al. Conventional and chest-compression-only cardiopulmonary resuscitation by bystanders for children who have out-of-hospital cardiac arrests: A prospective, nationwide, population-based cohort study. Lancet 2010; 375: 1347-1354.

14. Nadkarni VM, Larkin GL, Peberdy MA, Carey SM, Kaye W, Mancini ME, et al. First documented rhythm and clinical outcome from inhospital cardiac arrest among children and adults. JAMA 2006; 295: $50-57$. 